Cinémas

Revue d'études cinématographiques

Journal of Film Studies

\title{
Les lieux de la critique
}

\section{René Prédal}

Volume 6, numéro 2-3, printemps 1996

\section{La Critique cinématographique}

URI : https://id.erudit.org/iderudit/1000969ar

DOI : https://doi.org/10.7202/1000969ar

Aller au sommaire du numéro

\section{Éditeur(s)}

Cinémas

\section{ISSN}

1181-6945 (imprimé)

1705-6500 (numérique)

Découvrir la revue

\section{Citer cet article}

Prédal, R. (1996). Les lieux de la critique. Cinémas, 6(2-3), 11-28. https://doi.org/10.7202/1000969ar

\section{Résumé de l'article}

À partir d'un corpus français, l'auteur se propose de réfléchir à la nature et aux fonctions de la critique de cinéma s'exprimant dans les revues spécialisées ainsi que dans la grande presse et à la télévision : rôle d'information, d'expression, d'éducation..., mais pour quel type de communication? Quelle articulation avec théories et idéologies? Quel équilibre entre histoire, analyse et actualité? Hier organes des ciné-clubs et de la cinéphilie, à qui s'adressent aujourd'hui ces publications qui gagnent en légitimité à mesure qu'elles perdent de plus en plus leurs lecteurs? La crise actuelle est-elle due à la réduction de l'espace rédactionnel, à la faible crédibilité des textes, à la perte d'acuité du regard critique...? 


\section{Les lieux de la critique}

\section{René Prédal}

\section{RÉSUMÉ}

À partir d'un corpus français, l'auteur se propose de réfléchir à la nature et aux fonctions de la critique de cinéma s'exprimant dans les revues spécialisées ainsi que dans la grande presse et à la télévision: rôle d'information, d'expression, d'éducation..., mais pour quel type de communication? Quelle articulation avec théories et idéologies? Quel équilibre entre histoire, analyse et actualité? Hier organes des ciné-clubs et de la cinéphilie, à qui s'adressent aujourd'hui ces publications qui gagnent en légitimité à mesure qu'elles perdent de plus en plus leurs lecteurs? La crise actuelle est-elle due à la réduction de l'espace rédactionnel, à la faible crédibilité des textes, à la perte d'acuité du regard critique...?

\section{ABSTRACT}

Using French materials, the author offers a reflection on the nature and functions of film criticism in specialized magazines, as well as in mass publications and on television. What are the roles of information, expression, education, and for what type of communication? How is criticism articulated to theories and ideologies? What is the balance between history, analysis, and current issues? Formerly the organs of film clubs and cinephiles, who today is addressed by these publications that have gained legitimacy as they have lost readership? Is the present crisis due to a reduction of editorial space, a low credibility of the texts, or a loss of acuity in the critical gaze?

Cinéma et critique n'ont aucune matérialité: le premier, en effet, n'est même pas un métrage de pellicule, mais plutôt un 
reflet sur une toile le temps d'une projection; quant à la seconde, elle est une abstraction (un genre littéraire), une réflexion, c'està-dire une pensée. Support du texte critique, la revue matérialise donc de façon tangible des manifestations impalpables de l'esprit humain dont elle garde la trace en même temps qu'elle s'épuise à en apporter la preuve. À la fois mémoire vive et aiguillon de la création, la revue est un espace de débat, terre d'échange, mais aussi processus de légitimation artistique et relevé régulier des fièvres cinéphiliques.

Proposant quelques prolégomènes à une possible théorisation de l'étude de films, Pierre Baudry avance pour sa part l'idée que " [...] la critique a une fonction relativement analogue à celle des nouvelles boursières" (p. 10). De fait, les valeurs cotées sont les films, mais tout aussi bien les auteurs, les pays ou les genres: l'œuvre est introduite dans le concert cinéphilique à l'occasion d'une vision au cours d'un festival, son intérêt sera confirmé ou déjà revu à la baisse lors de la sortie parisienne et souvent réévalué lors de reprises ou de passages à la télévision bien des années après. Ne manque même pas dans les revues la cotation officielle pleine page ${ }^{1}$ où les principaux critiques notent tous les mois du point noir aux quatre étoiles les films retenus dans ce tableau d'honneur qu'il ne faudrait pas confondre avec les résultats du box office pieusement consignés dans l'organe professionnel $L e$ Film français. La revue qui publie ces cotations peut donc être assimilée à la bourse et il existe plusieurs revues de cinéma (Les Cahiers du cinéma, Positif, Sight and Sound, Film Quarterly, Cinema Nuovo ou Kinema Junpo) comme il y a plusieurs marchés boursiers (Paris, Londres, New York ou Tokyo). Les modes provoquent parfois des embellies sur les productions du tiers monde ou l'expérimental, mais les valeurs refuges (l'or ou le dollar du cinéma en quelque sorte) se recherchent vite, en période de crise, dans le cinéma états-unien!

Ces cotes sont établies d'ailleurs par un double système de comparaison horizontale - les films jugés les uns par rapport aux autres - et verticale en référence à des modèles explicites (avoués) ou implicites (voire pas toujours très conscients). Pierre Baudry parle de "[...] degré de conformité du produit à un modèle cinématographique, modèle dont la tendance est de res- 
ter perpétuellement non dit» et, plus loin, de "[...] l'exercice d'une conception du cinéma en conformité avec la politique générale du journal ou de la revue» (p. 10), laquelle est forcément l'émanation d'une idéologie dont le critique ne peut que tenir compte: se désintéresser de l'esprit du journal dans lequel il s'exprime serait en effet faire bien peu de cas de ses lecteurs! Mais il y a aussi les œuvres acratiques, selon la terminologie de Roland Barthes, c'est-à-dire qui ne peuvent pas être "traitées " par l'appareil critique habituel qu'elles mettent en crise au même titre que le système qui les a produites et qui se révèle aussi mal adapté à leur distribution qu’à leur exploitation.

\section{Les territoires critiques}

Pour Joël Magny, " [...] la critique n'a pas pour fonction première, ni même dernière, d'assurer le succès d'un film. Cela, c'est le rôle de la publicité et des attachés de presse" (p. 17). De fait, la critique n'est pas ce qui fait vendre, pas plus le quotidien où est imprimé le texte que l'œuvre dont il est question. Le travail d'analyse thématique et esthétique du critique se démarque donc radicalement de l'espace où s'édifient dossiers de presse (matière de base fournie aux rédactions sous forme de renseignements bio-filmographiques, entretiens, notes d'intention, etc.) et placards publicitaires (les plus efficaces possible pour attirer à tout prix le spectateur potentiel, ils viendront concurrencer l'argumentaire textuel du critique dans les colonnes voisines de son propre journal). Hors de - plutôt que systématiquement contre - ce domaine du promotionnel, l'expression critique peut prendre trois formes:

\begin{tabular}{|c|c|c|c|c|}
\hline & & critique & genre & écrit par \\
\hline Revues spécialis & & CINÉMA & littéraire (essai) & amateurs \\
\hline Grande presse & & explicative $\quad$ FILM & journalistique (article) ) & \\
\hline & masses-médias & & & professionnels \\
\hline Audiovisuel & & informative SPECTACLE & (intervention) $)$ & \\
\hline
\end{tabular}

Dans la grande presse s'inscrit la critique " [...] pour un jour, puissance rapide, passagère nulle et souveraine, destinée à introduire les œuvres sans précaution dans les remous et le cours du 
monde» (Blanchot, p. 141). Michel Ciment (p. 4-5) remarque qu'aujourd'hui, les journaux mettent plus volontiers en avant les chiffres d'entrées dans les salles que le texte du critique. Ainsi qu'il le note amèrement, le critique d'un journal comme $L e$ Nouvel Observateur disposait, dans les années 60, d'une double page pour parler du film qu'il voulait (même inconnu, en provenance du tiers monde ou à petit budget). Aujourd'hui, une telle liberté est devenue impensable dans le même magazine: il faut parler de ce qui fait déjà événement.

On est entré dans l'ère du clip critique: Pariscope définit en une cinquantaine de mots (dont la moitié pour le résumé du scénario) nature et valeur du film consciencieusement catalogué par genres. Il faut désigner, (re)connaître avant d'avoir vu, informer, communiquer et non goûter, savourer, réfléchir. C'est qu'aujourd'hui, les campagnes de marketing ont 10 fois plus d'ampleur qu'il y a quelques années et tendent à assurer le succès du produit en une ou deux semaines à peine, alors que l'action de la critique (en cela plus proche du bouche à oreille que du matraquage des médias audiovisuels) a besoin de plus de temps pour déplacer ses lecteurs. De plus, les journaux s'emplissent très souvent de potins ou de reportages, mais aussi d'entretiens sérieux et de matériel documentaire qui remplacent (ou limitent) les textes critiques en se présentant comme eux... l'esprit critique en moins. La couverture médiatique du Festival de Cannes est ainsi caractéristique: on y célèbre de plus en plus à grand fracas la messe du cinéma, mais on pratique de moins en moins l'analyse des films.

La critique journalistique des grands quotidiens et hebdomadaires réduit les hiérarchies en vantant aussi bien Leos Carax que Steven Spielberg, le film-œuvre (modèle éditorial) que le produit destiné au prime-time TV (modèle de flot). Par là se trouve conforté le mythe du cinéma unique à l'intérieur duquel Alain Cavalier et Claude Berri semblent mener côte à côte le même combat, celui du GATT et de l'" exception culturelle", alors qu'il y a véritable différence de nature entre Libera me (1993) et Germinal (1994). Néanmoins, force nous est de constater que cette attitude part généralement d'un bon sentiment. Le temps n'est plus en effet où le critique du Figaro ou de France 
Soir aimait sincèrement à la fois Les 400 Coups (François Truffaut, 1959) et La Vache et le Prisonnier (Henri Verneuil, 1959)! En 1995, les successeurs aux mêmes tribunes préferent Oublie-moi de Noémie Lvovsky avec Valeria Bruni Tedeschi à Un Indien dans la ville d'Hervé Palud avec Thierry Lhermitte. Mais ils savent aussi que leurs lecteurs sont plutôt attirés par le second film; ils ne sauraient donc en dire du mal. Mais la manière dont ils louangeront Oublie-moi tentera de convaincre ces lecteurs qu'ils pourraient aussi bien apprécier le premier long métrage de Noémie Lvovsky et, ne pouvant évidemment pas hausser Palud jusqu'au niveau de la jeune cinéaste, ils s'y emploieront en ramenant cette dernière à Un Indien dans la ville, afin de lui ôter toute étiquette "infamante" d'intellectualisme, de recherche, d'avant-gardisme, voire tout simplement de "jeune cinéma français", généralement dissuasive pour un public en quête de divertissement ou même de qualité...

Le temps et la place sont ce qui manque finalement le plus aux critiques de la grande presse, qui ont souvent la passion et la compétence nécessaires, mais ne disposent que de quelques lignes à écrire dans l'instant! La situation de leurs confrères de l'audiovisuel est, il est vrai, encore pire. Longtemps responsable du cinéma aux journaux télévisés de la chaîne généraliste France 3, Henry Chapier reconnaît en effet qu'il n'exerce pas vraiment sur le petit écran son métier de critique comme il le faisait au quotidien Combat dans les années 60 pour 30000 lecteurs. Là, pour 5 à 6 millions de téléspectateurs, Chapier déclare ne faire qu' " [...] un travail d'acteur qui n'a rien à voir avec la rédaction d'un papier» (Serceau, p. 167-168), tendant seulement par sa prestation de communiquer son enthousiasme pour un film. Devant les caméras vidéo, la redondance du métalangage s'installe effectivement aussi bien dans le discours des rhéteurs que dans les palabres du griot médiatique; c'est le retour du bonimenteur du cinéma des origines: parler sur le — ou à côté $\mathrm{du}-$ film.

Le niveau de tolérance de la télévision française à une émission critique sur le cinéma n'est guère élevé. Ainsi Le Masque et la Plume, grand classique de la radio où débattent depuis des années des critiques connus de la grande presse et des revues 
spécialisées, n’a tenu que quelques semaines à la télévision. De même, alors que revient enfin Cinéastes de notre temps, série mythique d'André S. Labarthe ressuscitée par la chaîne culturelle franco-allemande Arte, c'est aux dépens de la disparition de Cinéma-cinémas sur France 2, si bien que n'existe plus à présent aucune émission cinématographique critique sur une chaîne généraliste de grande écoute ${ }^{2}$. Sur Canal Plus, pourtant chaîne cryptée dite "du cinéma ", Le Journal du cinéma (hebdomadaire) n'est en effet qu'informatif, tout comme l'évocation une ou deux fois par semaine en fin du journal de 20 heures sur TF1 ou FR2 d'un film faisant sa sortie nationale à Paris: deux ou trois minutes avec un interprète ou le réalisateur (interviewés par le présentateur vedette n'ayant pas vu le film et ignorant tout du cinéma) suivies ou précédées d'un court extrait (fourni par le service de presse du film, c'est-à-dire toujours la même scène ou la bande annonce passant ainsi plusieurs fois sur les écrans)...

La revue de cinéma initiait dans les années 50 une approche du film d'une tout autre nature que celle proposée alors par le mouvement des ciné-clubs. Au contraire, dans leur très grande majorité ${ }^{3}$, les émissions de cinéma à la télévision n'ont pas donné naissance à de nouveaux moyens de lecture et d'étude du septième art. À l'inverse, elles ont même appauvri l'exégèse, car la parole est moins propice à l'analyse approfondie que la rédaction d'un texte. Or, il s'agit essentiellement de conversations de plateau ou de reportages de tournage réduisant la réalisation à ce seul moment spectaculaire, qui n'est pas forcément le plus créatif du triptyque écriture-tournage-montage. Seul l'entretien avec un cinéaste est peut-être plus révélateur à l'image que dans les pages d'une revue. Mais nous touchons là alors à la maladie endémique de toute la critique, écrite comme télévisuelle: elle fait trop parler les cinéastes au lieu de s'exprimer elle-même! Il y aurait pourtant moyen d'utiliser la spécificité des effets vidéo (recadrage, ralenti, arrêt sur image, remontage, rapprochement, etc.) pour mieux pénétrer la forme cinématographique et des collections utilisant ces procédés, destinées prioritairement aux élèves ou étudiants, existent même sur le marché en France depuis la diffusion en 1986 de l'analyse par l'image de $M$. le Maudit de Fritz Lang (1931): Jean Douchet y dissèque l'œuvre 


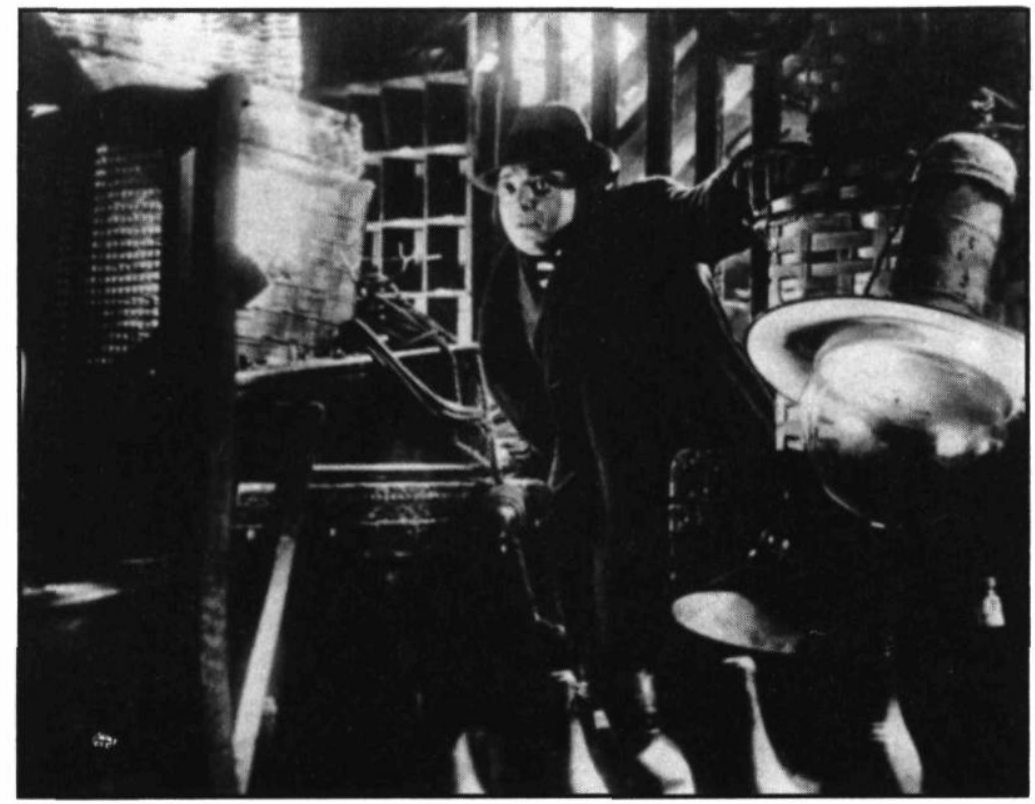

\section{Ie Maudit de Fritz Lang (1931)}

Collection Cinémathèque québécoise

avec pertinence à l'aide de déplacements ou superpositions soulignant le sens des raccords, la nécessité des mouvements, le jeu des éclairages en une sémiologie in vivo hélas jamais reprise dans les émissions télévisées, trop soucieuses d'en rester aux caractères généraux (la stylistique) au lieu de creuser la spécificité (à savoir l'esthétique). Seule la critique des revues spécialisées, expédiant en quelques lignes le tout venant des films du mois, se permet de cultiver la différence en se penchant longuement cette année sur Krzysztof Kieslowski, Atom Egoyan ou Abbas Kiarostami.

Flashback: les revues françaises

La critique de cinéma se dégage de la presse corporative (de type La Cinématographie française) dans les années 20 avec Louis Delluc et Cinéa, parallèlement à une prolifération de magazines grand public sur le cinéma, où l'essentiel des textes consiste en reportages, films racontés ou interviews de vedettes, souvent d'ailleurs non dénués d'intérêt cinéphilique comme dans Cinémonde créé en 1928. Avec La Revue du cinéma de Jean-George 
Auriol (lancée également en 1928), on approche davantage de la revue spécialisée d'aujourd'hui, mais les principaux périodiques critiques attendront la première décennie d'après-guerre pour se manifester. Naissent alors en effet: L'Écran français (1945), TéléCiné (1946), Image et Son (1946), Les Cahiers du cinéma (1951), Positif(1952) et Cinéma (1954).

Parmi ces revues, trois émanent du mouvement des ciné-clubs: Télé-ciné est l'organe de la fédération catholique, Image et Son, celui de la Ligue de l'enseignement (ancrée à gauche, laïque et à forte finalité pédagogique de culture populaire), Cinéma étant éditée par la Fédération française des ciné-clubs est idéologiquement et politiquement moins marquée. Et très vite s'affrontent deux politiques éditoriales aux Cahiers et à Positif: les premiers privilégient la notion de mise en scène tandis que le second s'attarde surtout aux sujets, ce qui conduit les Cahiers à préférer aux États-Unis Howard Hawks et Alfred Hitchcock tandis que Positif privilégie John Huston ou Richard Brooks. Surtout, les Cahiers dissertent sur les auteurs alors que Positif se penche davantage sur les films. En somme, on peut parler de critique à tendance matérialiste (Positif) et idéaliste (Cahiers) ou encore éthique et esthétique. Dès lors le clivage devient assez net et l'on rangera Présence du cinéma du côté des Cahiers, mais La Méthode ou Miroir du cinéma avec Positif. Les deux revues constituent donc deux pôles critiques, tendant à susciter des épigones et par rapport auxquels Cinéma et Image et Son se définissent en défendant aussi bien les auteurs Cahiers que Positif, tandis que TéléCiné pratique plutôt la soustraction en se limitant au cinéma qu'aiment aussi bien les deux revues de référence!

Dans les années 70 , toutes les revues sont plus ou moins gagnées par les idéologies, mais aussi par la théorie: Les Cahiers sont ainsi totalement engloutis par le marxisme maoïste et un jargon sémio-psychanalytique qui les mèneront très près de la disparition. Plus mesurée, l'irruption du politique est néanmoins notable dans les autres publications bien que l'expérimental d'un Jean-Marie Straub ou d'une Marguerite Duras soit également bien reçu et qu'une réaction cinéphilique se dessine avec la sortie en 1972 des premiers numéros d'Écran et de Cinématographe. 
Mais le début de la décennie 80 marque une entrée dans des temps difficiles. Si la diversification des loisirs et l'emprise de la télévision vont être fatales à la fréquentation cinématographique, le magnétoscope et l'effondrement du mouvement des ciné-clubs causent pour leur part le recul des revues spécialisées traditionnelles, mais la prospérité des magazines: Télé-Ciné avait déjà disparu en 1978, Écran en 1979; Cinématographe cesse en 1987; Cinéma tente de devenir hebdomadaire en 1985, mais ne sera plus qu'un minuscule bulletin de liaison; quant à Image et Son, après un changement de titre (Revue du cinéma), puis de structure (lâché par la Ligue de l'enseignement en 1990), il renaît un an et demi sous le nom de Mensuel du Cinéma avant de mourir définitivement en 1994. Malgré de délicats rééquilibrages financiers et quelques changements d'éditeurs, Les Cabiers du cinéma et Positif se partagent donc aujourd'hui la quasi-exclusivité d'un terrain critique désormais très consensuel, néanmoins enrichi par le lancement en janvier 1992 du trimestriel Trafic créé par Serge Daney.

\section{La double nature des revues de cinéma}

Si l'on veut dépasser l'antagonisme des deux écritures, celle du film et celle du texte, on peut classer les périodiques en cinq catégories:

- revues critiques: Cahiers du cinéma ou Séquences;

- magazines: luxueux (Studio) ou populaire (Première);

- publications thématiques (CinémAction) ou monographiques (Études cinématographiques);

- organes spécialisés (Le Technicien du film) ou professionnels (Filméchange);

- publications universitaires: Iris ou Cinémas.

La France est l'un des pays du monde où les revues sont, proportionnellement, les plus nombreuses. En principe, une revue est vendue en kiosque, mais Études cinématographiques figure en librairie et Iris ne se diffuse guère que par abonnements. Si leur économie est fragile, c'est que malgré une rédaction faisant largement appel au bénévolat (seule une poignée de critiques vit des textes publiés; personne n'est payé à Positif), les lois du marché s'appliquent sans concéder de privilèges tant dans la fabrication 
qua dans la diffusion des périodiques: un imprimeur facture son travail de la même manière à Positifou à Paris Match.

Or le lectorat n'est guère susceptible de s'accroître, car les cinéphiles les plus passionnés achètent déjà plusieurs publications. Mais si les revues de cinéma ne tirent qu'à quelques milliers d'exemplaires, ce n'est pas moins que les revues littéraires. Or, qui oserait affirmer que Tel quel ou Les Temps modernes n'ont aucune action! En fait, ces revues agissent en profondeur sur des couches particulières de lecteurs (étudiants, enseignants, animateurs culturels, etc.), qui jouent ensuite le rôle de relais pour diffuser autour d'eux les idées émises par les critiques.

Encyclopédie permanente du cinéma, la revue constitue d'abord, pour ceux qui la lisent, un signe d'appartenance. C'est le choix du cinéma en bibliothèque au lieu d'en vidéothèque, l'accroche à la façon galaxie Gutenberg du village global audiovisuel: la revue se collectionne, se garde et se regarde, se consulte grâce aux index; la collection acquiert de la valeur avec le temps, se réédite en volumes et transforme l'amateur de cinéma en cinéphile, c'est-à-dire avant tout en connaisseur. Aussi, le lecteur est-il généralement abonné, ce qui induit la notion de permanence, d'investissement à long terme. Pourtant, Serge Daney raconte comment, élève au lycée Voltaire, il allait au début de chaque mois coller son nez " [...] à la vitrine d'une modeste librairie de l'avenue de la République. Il suffisait que, sous la bande jaune, la photo noir et blanc de la couverture des Cahiers ait changé pour que le cœur me batte», car c'était signe que le nouveau numéro était paru; " [...] quant à l'idée de m'abonner, elle ne m'effleura jamais: j'aimais cette attente exaspérée" (p. 6). La revue suscite en effet un véritable fétichisme, lié à sa nature d'objet manipulable, et l'élégante couverture jaune en fit au moins autant pour la légitimation du cinéma que le contenu parfois incendiaire des articles de ses bouillants rédacteurs! Ainsi, le feu des révolutions artistiques brûlait sous le glacis distingué d'une présentation académique de bon aloi. Aujourd'hui, la couverture en papier d'emballage marron de Trafic joue évidemment sur un effet inverse: rudesse, sobriété, donc sérieux contre les joliesses - induisant l'idée de facilité - de la nouvelle maquette magazine des Cahiers. 
La revue impose en effet la mise en scène du discours critique - un texte et des photos -, c'est-à-dire sa mise en page (le papier davantage que les mots, la maquette), mais butera toujours sur la citation impossible à la différence des deuxpoints-ouvrez-les-guillemets de l'étude littéraire. Question de support, le film restera à jamais hors champ, quelque part hors d'atteinte, obligeant du même coup le discours critique à sortir aussi de lui-même — c'est-à-dire de sa nature littéraire — pour inventer des formes neuves d'appréhension comme d'expression. Les études cinématographiques constituent en effet par essence une terre de recherche et, en analysant le cinéma, elles fondent un moyen de communication qui se démarque de plus en plus des autres domaines de la critique d'art.

Pour ceux qui l'écrivent, la revue est en outre un espace pluriel, le pluralisme des opinions s'ajoutant à l'éclectisme de la matière (le sommaire composite) pour former un instrument pédagogique et/ou de connaissance. Le dictionnaire Larousse précise: "Critique: n.f. 1. Art d'expliquer et de juger les œuvres littéraires et artistiques. 2. Jugement porté sur une œuvre littéraire ou sur une œuvre d'art."

Le critique étant celui qui exerce la critique, nous retiendrons de cette définition plutôt expliquer que juger. En fait, le critique a préalablement une vocation d'amateur. On n'a pas vocation de critique de cinéma mais de cinéphile et l'on éprouve ensuite le besoin de faire partager la passion que l'on ressent pour l'objet de sa critique $^{4}$. C'est pourquoi le critique de revues spécialisées s'attache davantage à la dynamique de l'œuvre qu'aux éléments constitutifs de son équilibre: le moteur positif est toujours plus révélateur que les qualités "en creux" ordonnant l'ensemble. C'est pourquoi le critique doit être un spécialiste (qui ne prenne pas pour une nouveauté quelque pâle imitation), mais éclairé, ouvert sur l'émotion qui peut parfois être atteinte par des moyens très simples.

De toutes manières, les revues de cinéma ne sont pas des publications scientifiques mais des lieux d'expression personnelle où la subjectivité reste souveraine. Comme l'écrit Morvan Lebesque:

[...] nous faisons un métier de critique dans des journaux, les gens achètent ces journaux en partie pour nous lire. Eh bien, qu'est-ce qu'ils achètent? Pas notre 
infaillibilité, nous ne sommes pas infaillibles. Notre intelligence? Nous ne sommes pas plus intelligents que d'autres. Ils achètent notre sincérité (p. 5).

Le critique n'est donc pas un juge et la critique descriptive est finalement plus difficile à manier que la "critique couperet». Eric Rohmer rappelle que «Bazin pensait qu'il fallait raconter l'histoire». Mais il ajoute aussitôt :

[...] or il y a d'excellents articles qui ne racontent pas
l'histoire et parfois ne parlent même pas du film,
comme celui de Godard sur Amère Victoire qui est en
même temps un remarquable article sur Nicholas Ray
[...] ce qu'il faut essayer d'éviter, ce sont les articles sco-
laires: la forme, le fond, le pour, le contre, etc. Un bon
article, c'est un article qui donne libre cours à la per-
sonnalité (Carbonnier et Magny, p. 14).

D'ailleurs, où se situe le texte critique? Il est évident qu'il s'agit d'un produit hybride écartelé entre l'information (renseigner), l'expression (donner son avis, proposer son regard comme un créateur le fait dans son œuvre) et la communication (toucher un lectorat et influer sur ses choix en matière de spectacle).

En fait, le critique ne doit pas chercher à avoir raison pour l'éternité: il n'écrit pas pour la postérité mais dans le but d'agir sur son temps. Le critique est un spectateur qui sait; non pas qui sait obligatoirement ce qu'il faut penser de l'œuvre nouvelle qu'il vient de découvrir, mais qui sait un certain nombre de choses sur l'environnement culturel de cette production, ce qui lui permet au moins de la décrire correctement, et constitue déjà un pas décisif vers la compréhension de celle-ci, donc vers sa réception.

Pour Louis Séguin: "La critique est affaire de provocation plus que de conviction. Le meilleur rôle qu'elle puisse avoir est de susciter, à propos d'un film, des réactions, de préférence violentes, chez le lecteur" (p. 81).

En fait, la critique comme SAMU, selon le mot de Serge Toubiana (p. 21), est sans doute un leurre: ses "pin-pon" (on arrive pour "sauver" l'œuvre de l'indifférence, pour dire que c'est une grande création qu'il ne faut surtout pas rater) ne sont pas en effet d'une grande efficacité. On s'en est aperçu lors 
d'une fameuse polémique, en octobre 1982, où certains critiques essayèrent d'opposer Une chambre en ville, de Jacques Demy, à L'As des as, de Gérard Oury et Jean-Paul Belmondo, sorti la même semaine avec un énorme succès de tiroir-caisse. La défense (peut-être maladroite) du film de Demy, associée à l'éreintement de celui d'Oury, ne réussirent qu'à faire une publicité supplémentaire à ce dernier. Les médias de masse ouvrirent en effet largement leurs écrans ou leurs colonnes aux propos démagogiques de Belmondo qui répondait aux critiques en en appelant au public et en traînant dans la boue le cinéma d'auteur... cela sans ramener un spectateur de plus au film de Jacques Demy!

Aussi Serge Toubiana pense-t-il qu'il vaudrait mieux un critique passeur, c'est-à-dire jouant un rôle d'intermédiaire nécessaire entre l'œuvre et son destinataire, homme de goût traduisant le "message", décryptant le code secret pour ouvrir l'œuvre au public; fonction certes plus modeste, mais qui s'en tient à des paris plus judicieux.

\section{Un regard en crise}

Dans son numéro 13 (mars-avril 1955), Positif révèle au public, dans une étude signée Paul-Louis Thirard, le cinéaste Maurice Burnan. Dans la livraison suivante ( ${ }^{\text {os }} 14-15$, novembre 1955), plusieurs autres rédacteurs — dont Georges Sadoul et Jean Ferry - y vont à leur tour de leur article analytique pour le "dossier Burnan». En fait, il s'agit d'un savoureux canular, car Burnan n'a jamais existé, même si de nombreux cinéphiles se sont alors précipités à leur ciné-club préféré pour demander à voir les œuvres de ce fabuleux réalisateur occulté! Mais cette idée - jamais reprise depuis! - est typique d'une certaine époque où la critique savait être irrespectueuse et se prendre elle-même comme sujet d'ironie, où les revues s'amusaient avec les lecteurs sans se paralyser dans un esprit de sérieux trop souvent pédantesque. Il n'y a plus aujourd'hui de prime de plaisir à lire des revues de cinéma et cet esprit potache pourrait constituer une agréable antidote aux pesanteurs universitaires ${ }^{5}$.

Les principales revues naissaient alors pour prolonger par l'écrit l'effet de la double parole des ciné-clubs: présentation 
avant la projection puis débat ensuite. Mais au bout d'une dizaine d'années à peine, elles s'aperçoivent que leur action est par elle-même insuffisante et doit être complétée par deux fonctions supplémentaires, celles de programmation et d'édition. De fait, les revues parlent de certains films vus dans des festivals, mais distribués ni par le réseau commercial ni par celui des ciné-clubs. Elles décident donc de les montrer elles-mêmes en organisant à partir de 1966 des "Semaines" à Paris, où elles programment six ou sept œuvres qui deviennent ainsi emblématiques des goûts de la rédaction: Les Cahiers du cinéma lancent la mode, mais en 1967 s'ajoute la Semaine Midi-Minuit Fantastique suivie l'année après d'une manifestation similaire organisée par Positif et en 1969, par Cinéma. Dans un autre ordre d'idées, les revues touchent aussi durant la même période aux limites du "dossier" et décident d'élargir leur rôle en tant qu'ouvrages de référence en publiant des livres de synthèses qui veulent lutter contre le côté trop ponctuel de la critique d'actualité. Là encore, une revue inaugure la tendance: c'est Cinéma, en 1961, avec 20 ans de cinéma américain (Jean-Pierre Coursodon et Yves Boisset), réédité et complété par Bertrand Tavernier en 1970 puis en 1991. Image et Son et Les Cahiers du cinéma suivront, la première avec les Éditions Édilig et les seconds les Éditions de l'Étoile. "Semaines" et "Livres" témoignent de la vivacité des revues à s'adapter à des demandes latentes, quitte à se remettre radicalement en question. Hors du circuit commercial, elles ne peuvent en effet demander à la publicité de faire naître artificiellement dans le public le besoin auquel leur produit répondra. Héritières d'une vieille tradition humaniste de culture populaire, elles conservent donc le sens du service à rendre, la vocation de guide et d'éducateur.

En effet, qu'il suive aveuglément les avis de son critique favori, qu'il en prenne au contraire systématiquement le contre-pied ou qu'il le lise attentivement pour se forger son idée personnelle, le spectateur utilise la critique comme conseillère. Ira-t-il ou non voir le spectacle après la lecture de l'article? C'est un premier résultat. Certes, beaucoup ne lisent la critique qu'après le spectacle. Dans ce cas, au lieu de vouloir se faire une opinion, le lecteur cherchera à confirmer son propre sentiment en le confrontant - ou en l'opposant - à celui du critique. Le texte est 
donc à double détente: dès le premier paragraphe, il doit emporter l'adhésion (lecture rapide avant de décider d'aller ou non voir un spectacle); suivra l'argumentation pour convaincre un lecteur devenu spectateur. Le critique est par conséquent un spécialiste qui prend la peine d'expliquer, mais accepte aussi de s'exposer à des risques: est-il possible de réfléchir sur l'actualité, donc sans distance? Peut-on encore cultiver tout le cinéma mais rien que le cinéma ${ }^{6}$ ? Le prix à payer aujourd'hui (acquérir une cassette vierge sur laquelle on inscrira l'œuvre originale ou préférer un commentaire écrit sur l'œuvre explique en partie le déplacement progressif de la fonction "guide du spectateur" de la revue spécialisée vers la grande presse et parfois même jusqu'à la télévision ${ }^{8}$.

$\mathrm{Si}$ les fonctions de la revue spécialisée ont beaucoup varié selon les époques ${ }^{9}$ et le public visé ${ }^{10}$ demeure par contre l'action historique en profondeur sur le public. Or, en modifiant la réception (aval), elle a transformé peu à peu aussi la création (amont) : indiscutablement, le mensuel Les Cabiers du cinéma et l'hebdomadaire Arts ont préparé vers la fin des années 50 le bon accueil fait aux premiers films de la Nouvelle Vague en développant chez les cinéphiles l'attente d'un cinéma jeune, sorti des studios et dégagé des règles de la dramaturgie classique... que les critiques de ces deux publications (J. L. Godard, F. Truffaut, E. Rohmer, C. Chabrol, J. Rivette) allaient eux-mêmes combler en passant derrière la caméra pour incarner une politique des auteurs qu'ils avaient d'abord théorisée sur le papier!

Mais les revues françaises sont-elles encore des lieux de débats à l'heure où les oppositions entre Les Cabiers du cinéma et Positif s'estompent et tandis que disparaissent celles qui occupaient jadis le centre de cette opposition, en assurant la nécessaire tâche de vulgarisation intelligente? Le caractère éphémère, aléatoire, irrégulier des revues de cinéma, qui meurent sans cesse pour renaître aussitôt ailleurs et autrement, leur fragilité, leurs perpétuelles mutations formelles et idéologiques, leurs changements de format, de pagination ou d'éditeurs, la valse des collaborateurs et la précarité des rédacteurs en chef, leurs polémiques véhémentes ou consensus mous constituent en tous cas les ferments nécessaires à leur possibilité d'impact. Reflets pour les cinéastes de l'attente de ces spectateurs qu'ils ne rencontrent que 
trop rarement, les revues forment en effet des organismes vivants qui doivent bouger, la notion de mouvement étant inhérente à la réalité du cinéma et de son étude. Créateur et longtemps à la tête des Cahiers du cinéma, Jacques Doniol-Valcroze voyait la revue comme une "idée en marche", c'est-à-dire un projet engagé sur le front du cinéma. Envisagée de cette manière, elle est un outil qui a fait ses preuves et demeure irremplaçable.

\section{Université de Caen}

\section{NOTES}

1 Ainsi le célèbre "Conseil des 10 " s'installe pour la première fois dans Les Cahiers du cinéma $\mathrm{n}^{\circ} 52$ (1955) pour "[...] concilier la subjectivité des critiques (que l'on continuera de trouver d'ailleurs sous les signatures habituelles) avec l'exhaustivité et l'objectivité légitimement attendues par nos lecteurs" comme l'écrit la présentation du premier tableau signée "la rédaction". Il s'agit donc d'une information destinée à élargir le choix des collaborateurs réguliers des Cahiers en ajoutant aux avis des "critiques maison" (de manière générale 5 sur 10 des donneurs de conseils) ceux de personnalités extérieures à la revue: Georges Sadoul (à gauche) et Henri Agel (à droite) ou un producteur comme Pierre Braunberger. Au départ, la cotation va du point noir (inutile de se déranger) à xxx (à voir absolument), sigles auxquels viendront bientôt s'ajouter quatre étoiles pour les chefs-d'œuvre. Le Conseil des 10 sera supprimé en été 1968 (dernières cotations dans le $\mathrm{n}^{\circ} 202$ ), victime de la dérive vers le maoïsme culturel qui commence à séduire la rédaction dès les lendemains des événements de mai. Il ne reparaîtra plus aux Cahiers, mais des guides analogues fleurissaient alors dans d'autres revues où les étoiles brilleront plus longtemps. Ainsi Le Mensuel du cinéma pratiquait encore cet exercice avant sa disparition à la fin de l'année 1994.

2 Créé en 1982, Cinéma-cinémas reprenait comme générique celui du Mépris de Jean-Luc Godard. L'émission était produite par Anne Andreu, Michel Boujut et Claude Ventura, faisait appel à des critiques spécialisés et à des cinéastes, traitant du septième art à la manière de Positifou des Cahiers du cinéma. Peu à peu déplacée vers des créneaux horaires plus tardifs, elle a finalement été supprimée il y a deux ans. Cinéastes de notre temps d'André S. Labarthe et Janine Azin fut à peu près mensuelle de 1964 à 1972. Sa structure monographique la distingua d'entrée du patchwork de "petits sujets" caractéristique des magazines d'arts plastiques de l'époque. Il s'agissait donc de véritables films réalisés par de jeunes cinéastes sur des grands maîtres qu’ils admiraient, cela à une époque où le monopole étatique joint à la dictature syndicale interdisaient pourtant à tout réalisateur non habilité par l'ORTF de signer une émission... sauf sur ce no man's land culturel miraculeusement préservé. On se souvient ainsi par exemple de Jean Renoir le patron tourné par Jacques Rivette en 1966 ou du Robert Bresson de François Weyergans.

3 À l'exception, précisément, de Cinéastes de notre temps.

4 Dans son enquête sur le métier de critique, le numéro 301 de la revue Cinéma 84 demandait aux professionnels: "Comment êtes-vous venus à la critique de cinéma?" Les réponses sont intéressantes: 
— par la cinéphilie (uniquement) : $59 \%$;

— par le journalisme (vous étiez déjà journaliste) : $16 \%$;

- par la cinéphilie et le journalisme: $21 \%$.

5 N'oublions pas que la revue Positifavait été créée par des étudiants lyonnais préparant le concours d'entrée à l'École normale supérieure. Le gag Burnan est assez caractéristique du ton des plaisanteries de "classes prépa." des grandes écoles.

6 On peut se demander plus particulièrement pourquoi les revues de cinéma ne se sont jamais vraiment ouvertes à la télévision: quelques dossiers dans Télé-Ciné et Image et Son, des chroniques maigrichonnes - et deux numéros spéciaux - aux Cahiers du cinéma, strictement rien à Positif ne font pas en effet un bilan bien conséquent en 50 ans! Heureusement CinémAction fait depuis quelques années un très gros effort dans cette direction avec, en particulier, la publication depuis 1991 d'une Saison télévisuelle recensant et analysant toutes les émissions et téléfilms produits dans l'année.

7 Rappelons qu'avant l'époque du magnétoscope, le seul outil de référence était le film raconté (Ciné-Miroir de 1922 à 1953; Mon film de 1924 à 1959 et le Film complet de 1922 à 1959), puis le découpage plan par plan, établi à partir de la copie définitive étudiée à la table de montage, et rédigé dans un style lisible pour le profane (L'Avant-scène cinéma, créée en 1961, a publié à ce jour le découpage de 450 films).

8 Même si, nous l'avons montré plus haut, il ne s'agit plus dans ces masses-médias de critique mais seulement d'information.

9 Ainsi la volonté de faire reconnaître dans les années 50 le cinéma en tant qu'art majeur n'est-elle plus opératoire aujourd'hui où l'amour du cinéma et le désir de faire partager cet enthousiasme ne constituent plus sans doute des moteurs suffisants pour justifier l'existence d'une revue de cinéma.

10 En 1994, Le Mensuel du cinéma s'adresse à un public beaucoup plus large que Trafic, réservé à un petit nombre de cinéphiles confirmés.

\section{OUVRAGES CITÉS}

Baudry, Pierre. [Introduction à son analyse du dossier de presse de Tout va bien (JeanLuc Godard, 1972]. Cahiers du cinéma, n² 240 (juillet-août 1972).

Blanchot, Maurice. "La condition critique». Trafic, $\mathrm{n}^{\circ} 2$ (1992).

Daney, Serge. "Le travelling de Kapo". Trafic, $\mathrm{n}^{\circ} 4$ (1992).

Carbonnier, Alain et Joël Magny. "La critique en question ( $2^{\circ}$ partie). Entretiens". Cinéma 84, $\mathrm{n}^{\circ} 301$ (janvier 1984) p. 10-28.

Ciment, Michel. "De la critique dans tous ses états à l'état de la critique». Positif, n³ 313 (1987).

Lebesque, Morvan. "Débat ", dans "La critique". Cahiers du cinéma, n 126 (décembre 1961) p. 1-26.

Magny, Joël. "La critique en question ( $1^{\text {* }}$ partie). Flux et reflux ». Cinéma $83, \mathrm{n}^{\circ} 300$ (décembre 1983) p. 9-17.

Séguin, Louis. "Enquête", dans "La critique". Cahiers du cinéma, n 126 (décembre 1961) p. 48-84.

Serceau, Daniel. "Les émissions sur le cinéma à la télévision" (entretien avec Henry Chapier), dans "L'influence de la télévision sur le cinéma". CinémAction, n 44 (juin 1987) p. 166-168.

Toubiana, Serge. "Le point critique" [table ronde]. Cahiers du cinéma, n 356 (février 1984). 


\section{B IBLIOGRAPHIE}

"La critique". Cahiers du Cinéma, n 126 (décembre 1961).

"Le cinéma malade de la critique" (dossier réparti sur 6 numéros). Cinéma 66, $\mathrm{n}^{\text {os }} 102$ à 107 (janvier-juin 1966).

"Revues de cinéma ". Les cahiers du 7 art (nº́sécial), nº 2 (novembre 1986).

Andrew, Dudley. André Bazin. Paris: Éd. de l'Étoile, 1983.

Baecque, Antoine de. Cahiers du cinéma, histoire d'une revue. Tome 1: Les Cahiers à l'assaut du cinéma. Tome 2: Cinéma, tours détours. Paris: Éd. des Cahiers du cinéma, 1991.

Barrot, Olivier. «L'Écran français 1943-1953, histoire d'un journal et d'une époque ". Paris: Éd. Temps Actuels, 1979.

Bosseno, Christian (direction). "Le cinéma et la presse». La Revue du cinéma, du $\mathrm{n}^{\circ} 341$ (juillet 1979) au $\mathrm{n}^{\circ} 355$ (novembre 1980).

Gauteur, Claude. "Les revues de cinéma ". Magazine littéraire, n 36 (janvier 1970).

Guigon, Catherine. "Les revues de cinéma ". Presse Actualité, n 107 (février 1976).

Magny, Joël (direction). "La critique en question ". Cinéma, n 300 (décembre 1983) et 301 (janvier 1984).

Prédal, René. "Midi-minuit fantastique, étude analytique et sémiologique», 2 tomes. Université de Nice: Éd. du Centre du XXe siècle, 1977.

Prédal, René. "Un quart de siècle de "Cinéma" ". Cinéma 80 , nº spécial hors série.

Prédal, René. La Critique des spectacles. Paris: Éd. du Centre de formation et de perfectionnement des journalistes, 1988.

Toubiana, Serge (sondage sous la direction de). "Les français et le cinéma". Cahiers

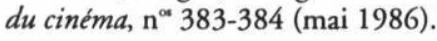

